\title{
Prosodic expression and levodopa in Parkinson's disease
}

\author{
Prosódia e levodopa da doença de Parkinson
}

Mara Behlau

PhD in Human Communication Disorders; Tenured Professor of the Postgraduate Program in Human Communication Disorders, at the Universidade Federal de São Paulo (UNIFESP); Head of "Centro de Estudos da Voz" (CEV), São Paulo SP, Brazil

Correspondence:

Mara Behlau

CEV

Rua Machado Bittencourt 361, 04044-001, São Paulo SP - Brazil.

E-mail:mbehlau@cevfono.com

Conflict of interest

There is no conflict of interest to declare.

Received 27 September 2013 Accepted 04 October 2013
$\mathrm{P}$ rosody is an important supra-segmental aspect of speech. Through prosodic variation, many cues regarding message intention, affected expression and type of interpersonal relationship are given to the listener. The main characteristics involved in prosody are fundamental frequency and loudness variability, speech rate, duration of pauses, vowel length, force of articulation and rhythm.

Prosody pattern is an individual characteristic that requires an intact central and peripheral nervous system. It is highly influenced by biological factors, cultural features, personality traits, emotional states and aging. It is easy to understand that many neurological and mental disorders can produce a direct impact on prosodic expression. For example, maniacal episodes are vocally characterized by uncontrolled intensity and excessive frequency variability; on the other hand, vocal fold paralysis can severely limit frequency and loudness control, impairing the speaking and singing voice.

Parkinson's disease (PD) patients suffer from reduction of the extent of all movements relating to speech, involving three subsystems: respiratory, phonatory and articulatory. In addition, swallowing may also be impaired ${ }^{1}$. Rigidity, bradykinesia and tremor are also present in oral communication, at several degrees of intensity that are not always directly related to the Hoehn and Yahr stages. PD patients' respiratory limitations do not provide proper breathing support for them to produce normal phrases and loudness variation; at the phonatory level, the vocal folds present reduced elongation, with restricted and unstable adduction impact on vocal quality and voice range profile; finally, at the articulatory level, speech sound imprecision and resonance imbalance contributes towards lower intelligibility scores. Patients with $\mathrm{PD}$ are usually characterized as presenting lower fundamental frequency (low pitch voice), monopitch and monoloudness (which gives the impression of a monotonous speech), phonoasthenia and lack of articulatory integrity ${ }^{1}$. The psychodynamics of individual with these characteristics are mostly negative: listeners may evaluate these patients as boring, unattractive and unhealthy, and even as having lower cognitive resources than their real condition permits $^{2,3}$. Moreover, since message intelligibility may be reduced, misinterpretations due to the lack of sound precision and reduced intonation patterns will possibly decrease communicative interactions, lead to greater social isolation and limit quality of life ${ }^{3}$.

Studies on voice and speech among PD patients, before and after treatment, frequently focus on the fundamental frequency and its variability and loudness levels in sustained vowels or selected phrases ${ }^{4,5}$. Within this context, the paper published in this issue of Arquivos de Neuro-Psiquiatria by Azevedo et al. ${ }^{6}$ adds an important attempt to assess prosody using several acoustic and temporal speech measurements. In addition to extracting 12 selected parameters relating to the oral communication impact of PD, the authors aimed to investigate differences in expressing attitudes (certainty and doubt) and in modes of speech (declarative and interrogative), which have singular melodic patterns. Ten individuals with idiopathic PD and ten individuals without neurological abnormalities (equal number of males and females) were analyzed. The PD patients recorded the speech material twice: firstly after twelve hours of abstention from use of levodopa (off) and then one hour after administration of the drug (on). The results were somewhat negative. As expected, lower values for the fundamental frequency occurred in the PD group and also the length values were higher in this group, probably due 
to bradykinesia and lack of respiratory support and control. Levodopa was important only for the durational parameters, which reinforces the idea that the drug only has a minor positive impact on speech. The surprising finding was that PD patients did not express attitudes or use modes of speech differently from the normal aging group. Methodological factors and the selection of the acoustic and durational parameters should be considered as potentially limiting factors.

Studies on prosody in these patients may have been neglected or avoided in the literature, probably due to difficulty in research design. The semi-automatic assessment that was performed, even with 12 selected parameters, did not reflect the social and clinical auditory impression of these patients' voices. Acoustic evaluation is a sophisticated puzzle composed by many individual parameters, while auditory analysis involves an evaluation on the overall perception. It has been well established that acoustic analysis frequently underestimates the overall auditory assessment ${ }^{7}$.

The study published in the present issue provides a contribution towards this subject but, in fact, to fully evaluate prosody, a multidimensional approach with combined measurements is required. Prosodic expression should not be defined as a summation of acoustic and durational parameters.

\section{References}

1. Sapir S, Ramig L, Fox C. Speech and swallowing disorders in Parkinson disease. Curr Opp Otolaryng Head Neck Sur 2008;1:205-210.

2. Behlau M, Madazio G, Azevedo R, Brasil O, Vilanova LC. Disfonais neurológicas. In: Behlau M (org.). Voz: o livro do especialista. Vol II. Rio de Janeiro, Revinter, 2005:111-154.

3. Ramig L, Fox C, Sapir S. Speech treatment for Parkinson's disease. Expert Rev Neurotherapeutics 2008:8:297-309.

4. Ramig L, Sapir S, Fox C, Countryman S. Changes in vocal loudness following intensive voice treatment (LSVT) in individuals with Parkinson's disease: a comparison with untreated patients and normal age-matched controls. Mov Disord 2001;16:79-83.

5. Constantinescu G, Theodoros D, Russell T, Ward E, Wilson S, Wootton R. Treating disordered speech and voice in Parkinson's disease online: a randomized controlled non-inferiority trial. Int J Lang Commun Disord 2011;46:1-16.

6. Azevedo LL, Reis CAC, Souza IS, Cardoso FEC. Prosody and levodopa in Parkinson's disease. Arq Neuropsiquiatr 2013;71:835-840.

7. Roy N, Barkmeier-Kraemer J, Eadie T, et al. Evidence-based clinical voice assessment: A systematic review. Amer J Speech Lang Pathol 2013;22:212-226. 